\title{
Computationally Assisted STEM and EXAFS Characterization of Tunable Rh/Au and Rh/Ag Bimetallic Nanoparticle Catalysts
}

Stephen D. House ${ }^{1}$, Cecile S. Bonifacio ${ }^{1}$, Janis Timoshenko ${ }^{2}$, Pranaw Kunal ${ }^{3}$, Haiqin Wan ${ }^{3}$, Zhiyao Duan $^{3}$, Hao Li ${ }^{3}$, Judith C. Yang ${ }^{1}$, Anatoly I. Frenkel ${ }^{2,4}$, Simon M. Humphrey ${ }^{3}$, Richard M. Crooks ${ }^{3}$, and Graeme A. Henkelman ${ }^{3}$

1. Dept. of Chemical and Petroleum Engineering, University of Pittsburgh, Pittsburgh, PA (USA)

2. Dept. of Materials Science and Chemical Eng., Stony Brook University, Stony Brook, NY (USA)

3. Dept. of Chemistry, The University of Texas at Austin, Austin, TX (USA)

4. Chemistry Division, Brookhaven National Laboratory, Upton, NY (USA)

The acceleration of rational catalyst design by computational simulations is only practical if the theoretical structures identified can be synthesized and experimentally verified. Of particular interest are bi-functional/bimetallic catalysts, which can have the potential to exceed the selectivity and efficiency of a single-component system [1]. However, adding a second metal greatly increases the complexity of the system; variation in the elements' mixing patterns and reconfiguration can affect the reaction mechanisms and thus catalytic performance [2].

Most experimental tools for the characterization of nanoparticles (NPs) provide structural data at the relevant length scales, but not enough to unambiguously determine the structure. Extended X-ray absorption fine structure (EXAFS) spectroscopy samples particle ensembles to determine the average coordination number, interatomic distance, and disorder through fitting to a reference structure [3]. Scanning transmission electron microscopy (S/TEM) locally characterizes materials down to the atomic scale and can measure the particle size, morphology, and elemental distributions necessary to inform accurate input models for interpreting the EXAFS data [4]. Correlating the local characterization of TEM with the many-particle information from EXAFS, grants a multiscale understanding not achievable with either approach alone. Here we present our application of this correlative theory-experiment design approach to characterize $\mathrm{Rh} / \mathrm{Au}$ and $\mathrm{Rh} / \mathrm{Ag}$ bimetallic hydrogenation catalysts, which our calculations predict to have superior allyl alcohol hydrogenation performance compared to single-element catalysts due to the ability to tune the hydrogen binding on the surface. [5].

The NPs were synthesized via microwave heating (MARS 5, CEM Corp.) using previously reported methods [5]; however, metal precursors were added only once. EXAFS characterization was carried out at the Stanford synchrotron radiation lightsource (SSRL). Small metal NP structures were calculated by density functional theory (DFT) and interatomic potentials under the frame work the modified embedded-atom method (MEAM) were employed to simulate EXAFS spectra. STEM imaging, energydispersive X-ray spectroscopy (EDS), and in situ heating were performed using an FEI TitanX ChemiSTEM, an FEI Talos F200X with Super-X EDS, and an FEI Themis 300 with Super-X EDS.

$\mathrm{Rh} / \mathrm{Au}$ and $\mathrm{Rh} / \mathrm{Ag}$ bimetallic NPs of different metal mixing ratios (and their monometallic constituents) were studied under a range of experimental conditions (air and $\mathrm{H}_{2}$-rich atmospheres; room, $\mathrm{LN}_{2}$, and $400{ }^{\circ} \mathrm{C}$ temperatures) using synchrotron EXAFS. MEAM potentials for Au-Rh and Ag-Rh were developed - and validated by reproducing $\mathrm{Au} / \mathrm{Rh}$ and $\mathrm{Ag} / \mathrm{Rh}$ foil spectra - for simulating the EXAFS spectra of the Rh/Au and Rh/Aa bimetallic NPs. No single simple model - random alloy, partially segregated, or phase segregated - fully reproduced the experimental data (e.g., Figure 1). STEM 
imaging and EDS mapping observations of the synthesized $\mathrm{Rh} / \mathrm{Au}$ samples revealed heterogeneous, multimodal morphologies: larger ( 3-10 nm), core-shell-like Rh/Au NPs (Au@Rh); smaller (1-3 nm) unalloyed Rh-only NPs; and a Rh-rich background of sub-nm clusters and non-associated individual atoms (See Figure 2a-b). Both the nanoparticle size and relative abundance of particle type depended on the stoichiometry. The morphology of the core-shell particles also varied with stoichiometry, with $\mathrm{Rh}$ in the 50:50 mixture showing a greater tendency for preferential attachment to certain facets of the $\mathrm{Au}$ cores than in the 90:10 mixture (e.g., Figure 2b-c). The Rh/Ag samples were found to possess a similar structural heterogeneity as the $\mathrm{Rh} / \mathrm{Au}$, though the "background" was Ag-rich. These TEM results provided the necessary information to develop the more accurate heterogeneous model needed to interpret the EXAFS data. Preliminary testing of this new model shows better agreement with the experimental spectra and DFT. [6]

References:

[1] A.K. Singh and Q. Xu, ChemCatChem 5 (2013), p. 4754-4766.

[2] R. Ferrando, J. Jellinek, R.L. Johnston, Chem. Rev. 108 (2008), p. 845-910.

[3] A.I. Frenkel, Chem. Soc. Rev. 41 (2012), p. 8163-8178.

[4] J.C. Yang, et al., Chem. Soc. Rev. 41 (2012), p. 8179-8194.

[5] S. Garcia, et al., ACS Nano 8 (2014), p. 11512-11521.

[6] This work was supported by NSF DMREF through contract No CHE-1534630. The authors would like to acknowledge FEI Company for additional microscopy work.
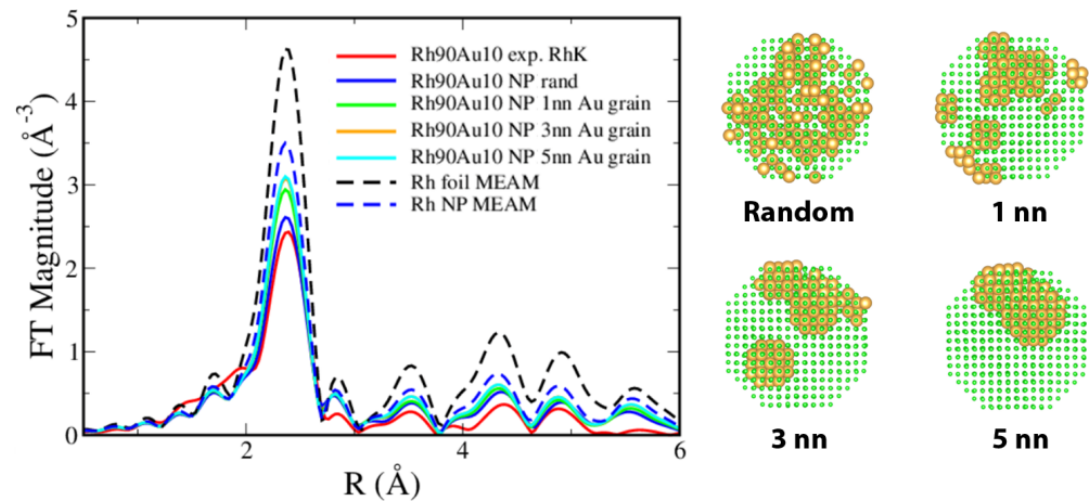

Figure 1. Experimental and simulated Rh K-edge EXAFS at $298 \mathrm{~K}$ for Rh90Au10 for different Au grain sizes; the K-edge height discrepancies arise from the use of an oversimplified input model.
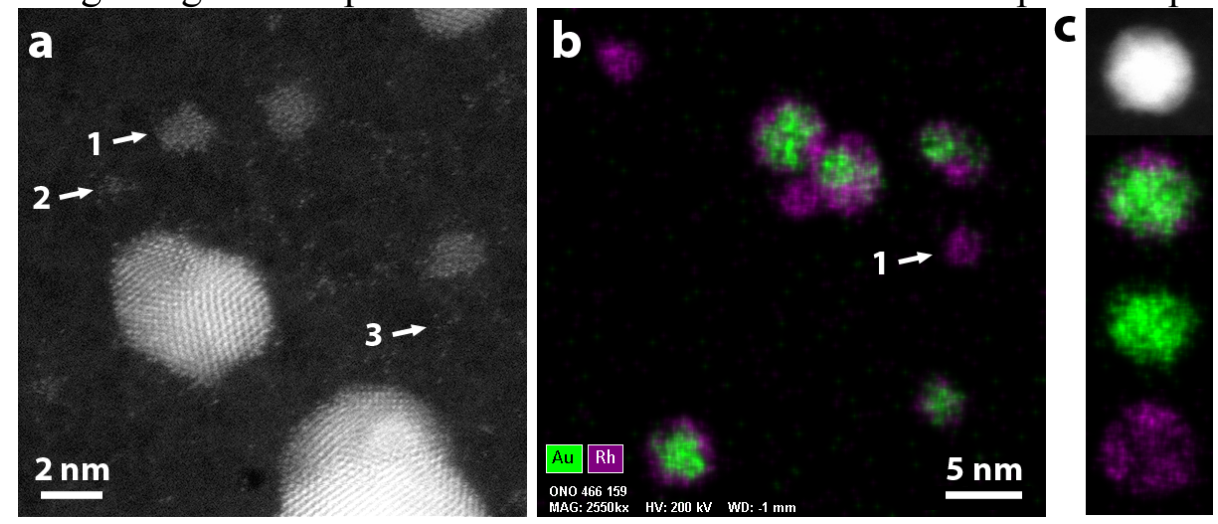

Figure 2. (a) HAADF-STEM image from the Rh90Au10 sample, showing larger, mixed Rh-Au NPs, (1) smaller Rh-only NPs, and Rh-rich (2) ultrasmall clusters and (3) non-associated atoms. (b) EDS map from the Rh50Au50 specimen showing the core-shell structure of the mixed particles; Au is green, $\mathrm{Rh}$ is purple. (c) A clear example of preferential Rh-attachment to certain facets of the Au core. 